\title{
Characterization of network harmonic impedance for grid connection studies of renewable plants
}

\author{
P. Eguia ${ }^{1}$, G. Gil ${ }^{1}$, R. Rodriguez-Sanchez ${ }^{2}$, M. Haro-Larrode ${ }^{2}$, A. Gil-de-Muro ${ }^{2}$ \\ ${ }^{1}$ Department of Electrical Engineering \\ E.I. Bilbao, University of the Basque Country UPV/EHU \\ Alda. Urquijo s/n, 48013 Bilbao (Spain) \\ Phone/Fax number:+0034 946 014970, e-mail: pablo.eguia@ehu.eus \\ ${ }^{2}$ Tecnalia \\ Parque Científico y Tecnológico de Bizkaia - Geldo, Edificio 700, 48160 Derio (Spain) \\ Phone+0034946430069, e-mail: raul.rodriguez@ tecnalia.com
}

\begin{abstract}
The impedance of a grid as a function of the frequency, or grid harmonic impedance, indicates the nature of the distortion and, especially, of the resonances that may appear in the system due to the presence of harmonic sources. Modelling this impedance is fundamental to be able to perform grid connection studies of renewable generation plants. However, usually the necessary information is not available, and the impedance is represented in a very simplified way. This paper analyses the different methods proposed in the literature to model the grid harmonic impedance and selects the most suitable for its implementation in PowerFactory simulation software, widely used in the industry for this type of studies. The selected method allows obtaining an equivalent network model that reproduces the harmonic behaviour of the network, without having to model the complete network in detail. A case study that applies the method to analyse the harmonic distortion at the POI of an offshore wind farm is presented.
\end{abstract}

\section{Key words}

Harmonic impedance, resonance, interconnection study, offshore wind power, PowerFactory.

\section{Introduction}

The presence of harmonics is one of the main sources of distortion in the voltage waveform. When a new renewable generation plant project submits a petition for connection to the network, one of the studies needed is a harmonic analysis. This is to ensure that the connection requirements established in the grid codes can be met by the plant [1].

The existence of harmonic currents has an associated series of negative consequences, such as heating of conductors, reduction in the transmission capacity of lines, overloading of transformers, etc. [2]. Additionally, the problem of interaction between the generation plant and the network at certain frequencies arises. If the impedance of the network has a large value for a certain frequency, harmful harmonic voltages may appear if the generator injects current at this frequency, while a low impedance value for a certain frequency may give rise to excessive currents if the generator voltage has a voltage harmonic at the same frequency. Therefore, knowing the impedance profile of the network as a function of the frequency is necessary when performing harmonic studies.

In order to know the grid harmonic impedance at the Point Of Interconnection (POI), it is necessary to know the complete structure of the network. However, this information belongs to the network operator and it is not in the public domain. Even if this information is known, modelling the network in such detail complicates the interconnection studies. Therefore, it is advisable to have a simplified grid model that represents, with enough precision, the real harmonic impedance profile of the network. This model can be created from the impedance vs. frequency plot at the POI, which, in turn, can be calculated by the network operator with a frequency sweep study.

Thus, this article analyses different methodologies proposed in the literature to represent the harmonic impedance of the network. The objective is to determine the methodology that can be implemented, in a simple way, in PowerFactory software and that represents the harmonic impedance of the network with enough accuracy.

The paper is structured in five chapters including this introduction. Chapter 2 introduces the concept of grid harmonic impedance and Chapter 3 describes different methodologies that reduce the grid to a simple network model. Once the most appropriate methodology has been determined, Chapter 4 describes its application to a case study involving the connection of an offshore wind farm 
to a large grid. The results obtained with both the equivalent and the complete network models are compared. Finally, chapter 5 contains the main conclusions

\section{Harmonic impedance of a grid}

A network can be characterized for harmonic studies with two parameters: the ambient or background distortion, which represents the harmonic distortion present at the POI without the generation plant connected; and the grid harmonic impedance.

The background distortion is defined by the voltage magnitude of each harmonic order present at the POI. It varies depending on the harmonics emitted by other sources connected to the network and due to changes in the network topology. Background distortion is usually not available, although it can be obtained by measurements during long time periods [3]. This information can be used to create a model, as in [4], where a methodology is proposed to represent the time-varying nature of the background distortion using time slicing and probability statistics techniques. A simplifying approach proposed in [2] consists in using the voltage distortion limits contained in the standards [5]-[6] or in the planning procedures used by the network operator. However, this approach does not guarantee that actual values comply with the limits and it could lead to unrealistic results.

The grid harmonic impedance usually is represented using a Bode plot, Figure 1, where the impedance magnitude and phase in the $y$-axis are given for each frequency value, or harmonic order, in the x-axis. A peak in the impedance magnitude represents a parallel resonance, while a valley represents a series resonant frequency.

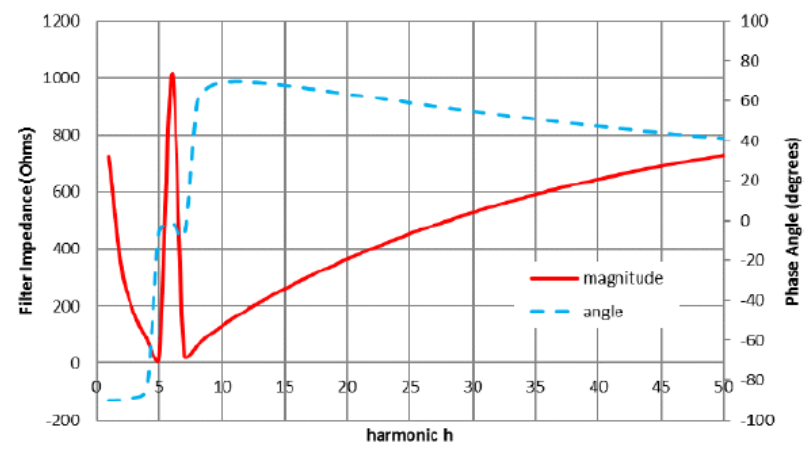

Fig. 1. Bode plot representation

The Bode plot of the impedance at the POI is not unique. Similarly to what happens with the background distortion, the grid harmonic impedance changes with the configuration of the grid and the connection state of the reactive power compensation equipment, generators and loads. This change in the topology can lead to major differences in the resonant frequencies. To account for this variation, authors in [2] propose to define a range between a maximum and a minimum value for the module and phase of the impedance for each frequency (Figure 2). This range should consider all possible contingencies and operating conditions of the network.
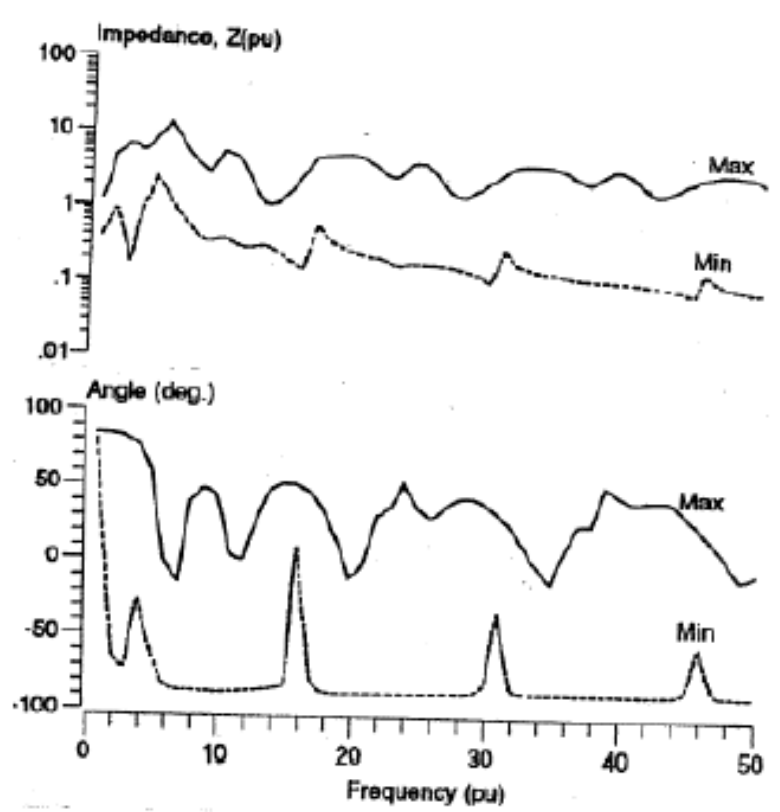

Fig. 2. Bode diagram with max/min envelope representation [2]

It is also possible to represent the grid harmonic impedance with values in the R-X plane for each specific harmonic frequency (Figure 3), where each point corresponds to a specific operating condition [2]. The curve envelop defines the boundary of maximum and minimum impedance values.

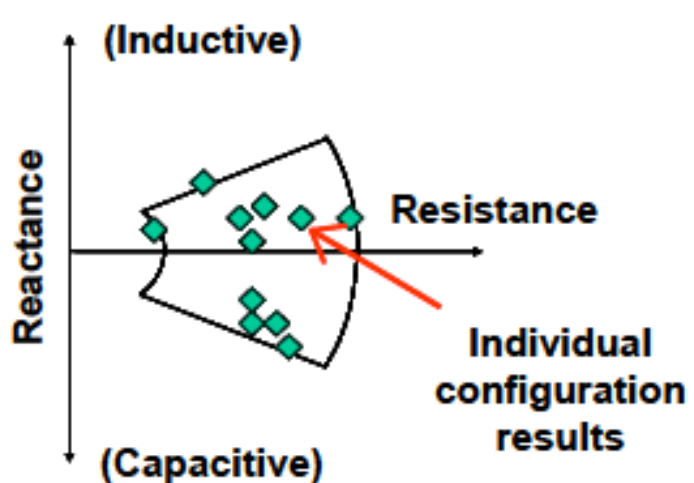

Fig. 3. Impedance representation for a single frequency [2]

Extending the previous approach to different harmonic frequencies [7], it is possible to obtain a representation similar to the one shown in Figure 4.

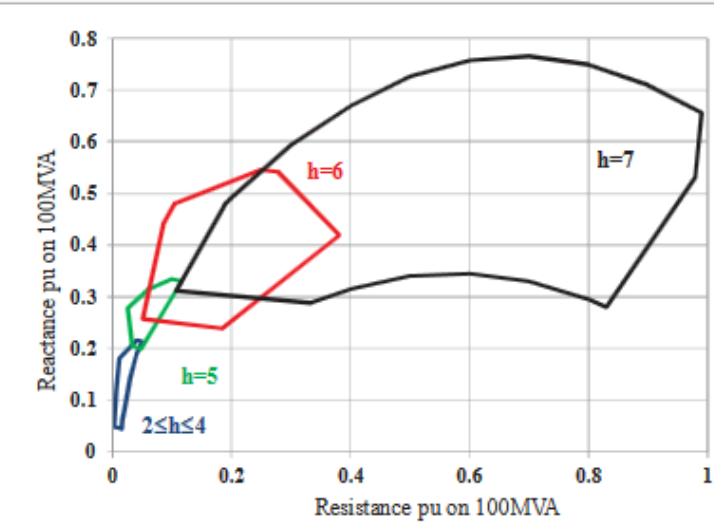

Fig. 4. Boundary impedance representation for different harmonic frequencies [7] 
The harmonic impedance of a network, as seen from the POI, can be obtained from measurements [3] or by calculation, using a frequency sweep tool applied to a network model. Either procedure will provide a set of impedance vs frequency curves that represent the harmonic response of the network. This information provides the starting point for the modelling methodologies explained in the following chapter.

\section{Comparison of methodologies for modelling the grid harmonic impedance}

When it comes to obtaining reliable results from a harmonics study for the connection of renewable generation, it is necessary to have a complete model of the grid. From this model, it is possible to build a Frequency Dependent Network Equivalent model (FDNE) using a conversion procedure [8]. These models are usually available in Electromagnetic Transient (EMT) programs. However, network information is usually only available to the network operator, so it is necessary to have an alternative procedure.

The simplest way to share network characteristics is based on the information of the impedance vs frequency curve, or the set of limit curves. These curves can be supplied by the network operator. With this information, the grid can be modelled either with one impedance, or with a set of impedances, connected to the POI. The advantage of this approach is that it can be implemented directly in any power system analysis software.

Bellow, three methodologies to represent a network through impedances are analysed and compared: short circuit impedance, RL//C filter and RLC branches in parallel. From the first to the latter, complexity and accuracy increases.

The comparison is carried out using a small distribution network as case study. The network, shown in Figure 5 with the POI highlighted in red, is part of an example grid taken from the power system analysis software PowerFactory [9]. The three models developed in line with these methodologies have been implemented in this software, for easy comparison.

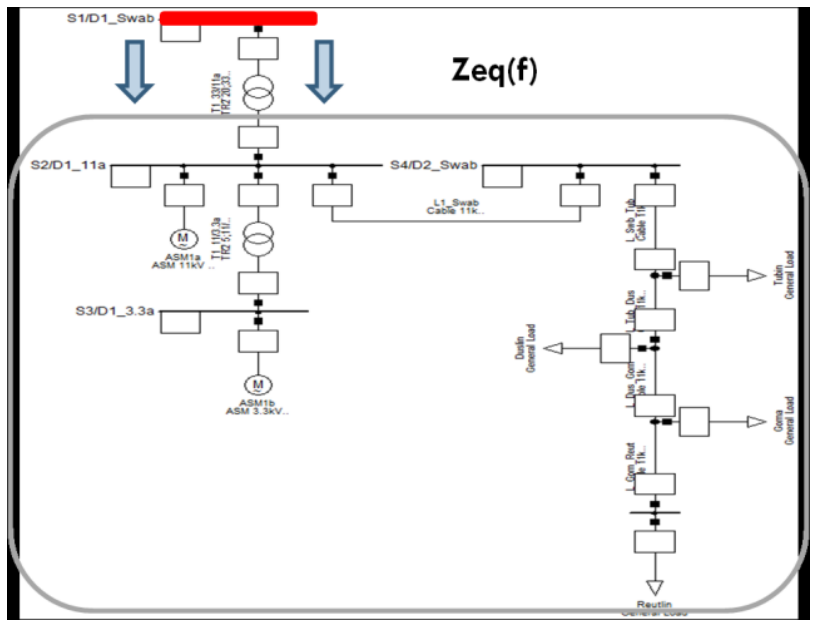

Fig. 5. Distribution network model for comparison of methodologies
The harmonic impedance of the case study network, seen from the POI, has been obtained with a frequency sweep analysis. The impedance bode plot is shown in Figure 6 . The grid impedance presents a parallel resonance for 755 $\mathrm{Hz}$ and series resonances for 0 and $900 \mathrm{~Hz}$.

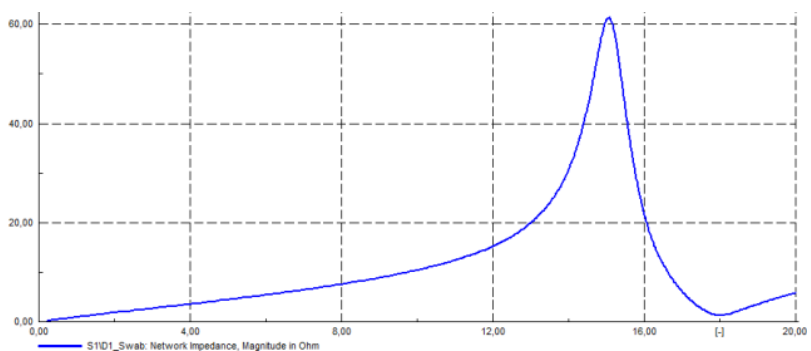

Fig. 6. Impedance vs. frequency at the POI

The following subsections show the results of applying each of the three methodologies and their comparison with the impedance profile of the reference network.

\section{A. Short circuit power model}

Traditionally, a network has been represented by a Thevenin equivalent, consisting on a voltage source and a RL impedance in series. These parameters are easily calculated from the voltage level and the short circuit power at the POI.

For the previous grid, the short circuit power at the POI $(33 \mathrm{kV})$ is $1096 \mathrm{MVA}$ with an $\mathrm{R} / \mathrm{X}$ ratio of 0.3 . This translates into an impedance equal to $0.9936 \Omega$ with $0.2855 \Omega$ resistance and $0.9517 \Omega$ reactance. However, the available information corresponds to the fundamental frequency, so this model will match the behaviour of the grid only around this frequency [2].

This can be clearly seen in Figure 7, where the frequency response of the short-circuit impedance model (red) is compared with the response of the reference network (blue). For low order harmonics, the accuracy of the model is adequate, but for higher frequencies the error is very large.

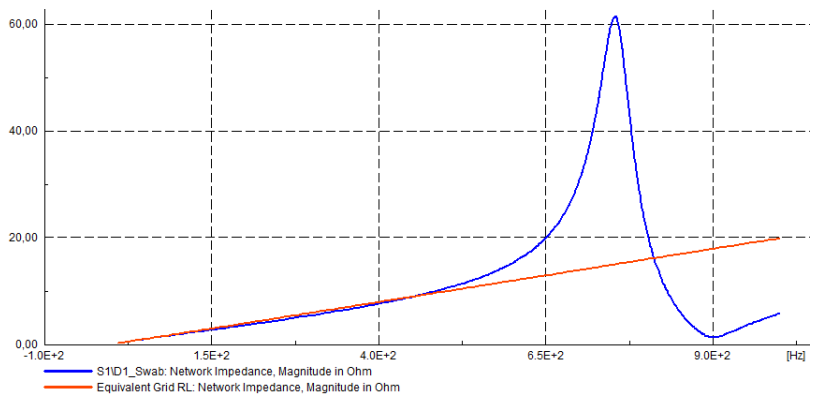

Fig. 7. Comparison short circuit power model

\section{B. $\quad$ RL//C filter tuning}

Due to the limitations of the previous model, some authors proposed a detailed representation of one or more nodes near the bus of interest, replacing the rest of the system with a Thevenin equivalent. An illustrative example of this line of research can be found in [6]. However, the accuracy 
of the frequency response depends strongly on the size of the network. For small networks, multi-node representation is sufficient, but in larger networks it is necessary to consider all the nodes of the system to achieve reliable results.

To try to keep the equivalent model simple with a good precision, this methodology proposes to characterize the network by an RL branch in parallel with a $\mathrm{C}$. The values of the parameters of the model can be obtained by an adjustment to the desired impedance profile.

For the example, the original impedance vs frequency curve has been fitted to the impedance of the filter, which corresponds to equation (1).

$$
|Z(f)|=\sqrt{\frac{R^{2}+(2 \pi f)^{2}}{1+16 C^{2} f^{4} L^{2} \pi^{2}-2 C L+C^{2} R^{2}}}
$$

The parameters of the filter that minimize the root mean square error $(\mathrm{RMSE})$ are $\mathrm{R}=0.251282 \Omega, \mathrm{L}=0.8535347$ $\mathrm{mH}$ and $\mathrm{C}=54.6293 \mu \mathrm{F}$.

Substituting in the example the complete network with a voltage source in parallel with the RL//C filter provides the frequency response shown in Figure 8 in the green curve.

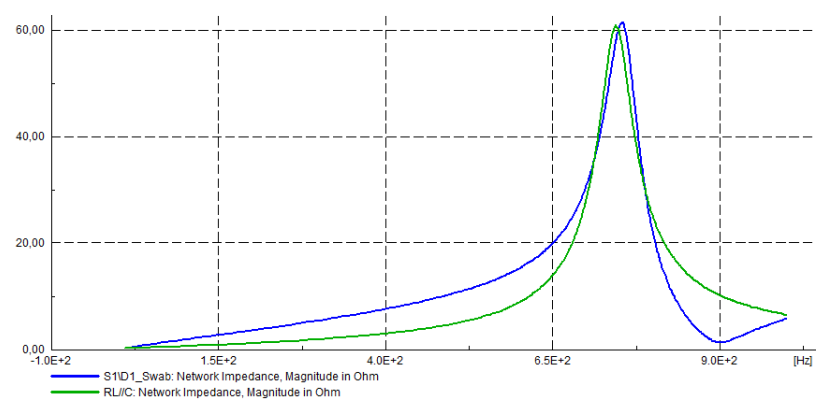

Fig. 8. Comparison of RL//C filter model

Comparing the impedance plot with the original one, this method provides good precision to characterize the parallel resonance, but the precision is lost outside this frequency.

\section{Parallel RLC branches}

The third methodology is based on the work carried out by Hingorani, Narain and Burbery [10]. The method consists of representing the network at the POI with a set of RLC branches. The methodology is simple to implement, and the model obtained can be included in any network simulation program.

Given a network whose harmonic impedance profile presents $\mathrm{n}$ resonances in series and $\mathrm{n}-1$ resonances in parallel, the grid equivalent model will be formed by $n$ branches in parallel connected to a voltage source. Each branch is formed composed of an inductance, a resistor and a capacitor connected in series, except for the first branch that does not include a capacitor.

For the example of the previous network, the grid impedance presents a series resonance for $0 \mathrm{~Hz}$ and $900 \mathrm{~Hz}$ and a parallel resonance for $755 \mathrm{~Hz}$. Therefore, it can be modelled with two RLC branches. Applying the methodology described in [10] the results shown in Table I are obtained.

Table I. - RLC branch parameters

\begin{tabular}{|l|c|c|}
\hline & \multicolumn{2}{|c|}{ Branch number } \\
\hline & 1 & 2 \\
\hline $\mathrm{R}(\Omega)$ & 1 & 1.2804 \\
\hline $\mathrm{L}(\mathrm{mH})$ & 2.5375 & 6.0274 \\
\hline $\mathrm{C}(\mu \mathrm{F})$ & & 5.1883 \\
\hline
\end{tabular}

The impedance profile obtained with this model (red curve of Figure 9) adjusts with the best precision to the impedance profile of the original network, compared to the previous methodologies.

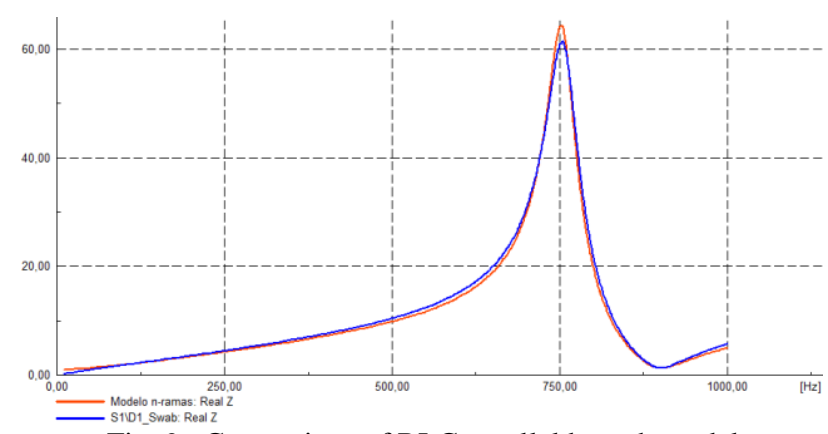

Fig. 9. Comparison of RLC parallel branch model

\section{Case study}

In the previous chapter, it has been demonstrated that the methodology proposed by Hingorani, Narain and Burbery allows to obtain an equivalent model of a network that represents with high precision the harmonic impedance of the network.

In this section, the application of the RLC parallel branch methodology is analysed for a study of the interconnection of an offshore wind farm to a large AC network through an HVDC link [11]. Figure 10 shows the windfarm internal grid and the HVDC link and Figure 11 the external AC grid with the POI (green square).

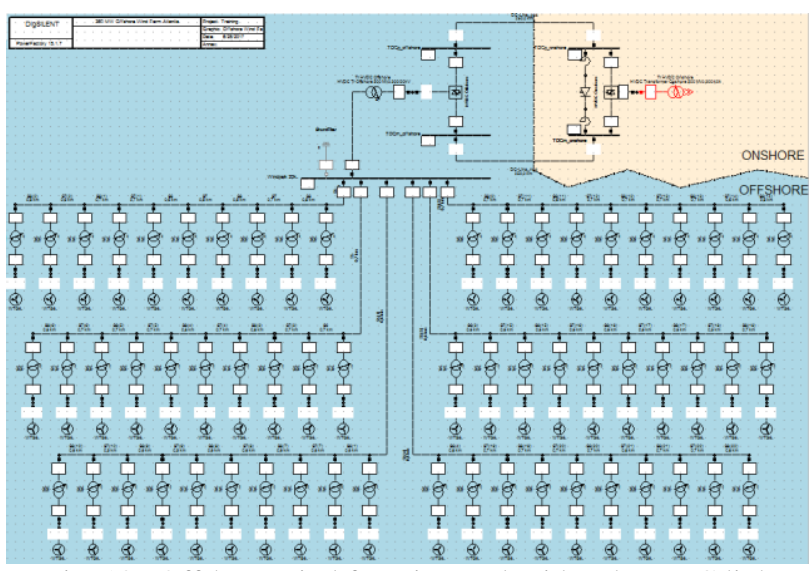

Fig. 10. Offshore wind farm internal grid and HVDC link

The external grid is a $400 \mathrm{kV}$-meshed network composed by 17 substations, 29 lines, 7 generating stations, 11 equivalent loads and 5 reactive compensation devices. 


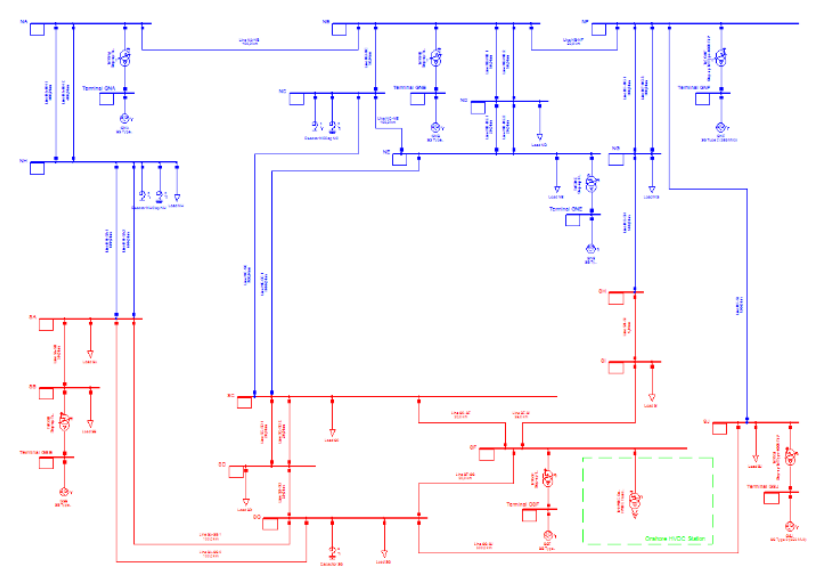

Fig. 11. External AC grid

Depending on the configuration of the different equipment, and on the switching state of the lines and compensation equipment, the grid impedance seen from POI will change. Figure 12 shows the grid impedance profile as a function of the frequency as seen from the POI, for the grid configuration shown in Figure 11.

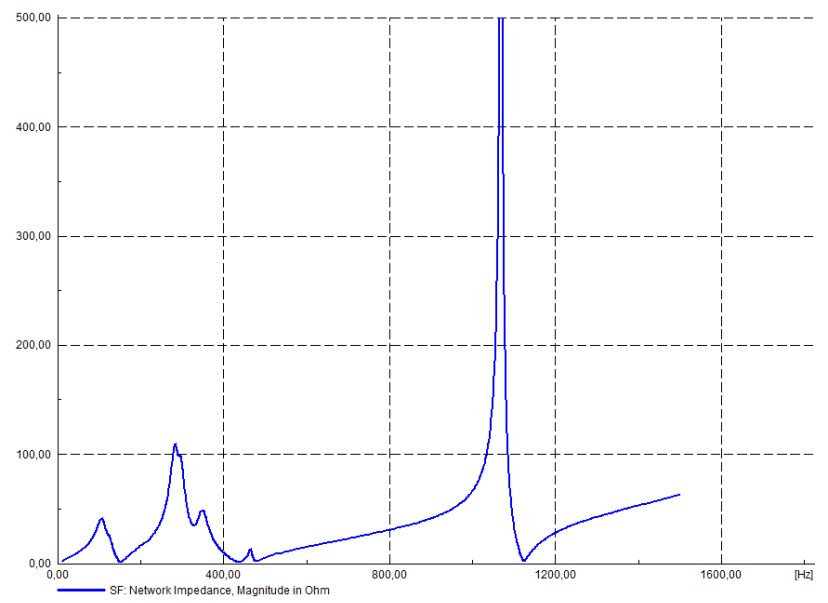

Fig. 12. Impedance vs. frequency at the POI

The network impedance presents 6 series resonances $(0 \mathrm{~Hz}$, $152.5 \mathrm{~Hz}, 328.5 \mathrm{~Hz}, 436 \mathrm{~Hz}, 479.4 \mathrm{~Hz}$ and $1125 \mathrm{~Hz}$ ) and 5 parallel resonances $(106 \mathrm{~Hz}, 283.4 \mathrm{~Hz}, 349.3 \mathrm{~Hz}, 465.5 \mathrm{~Hz}$ and $1069 \mathrm{~Hz}$ ). Applying the methodology of the RLC branches in parallel, the grid at the POI can be represented by a voltage source with 6 RLC branches in parallel, as shown in Figure 13.

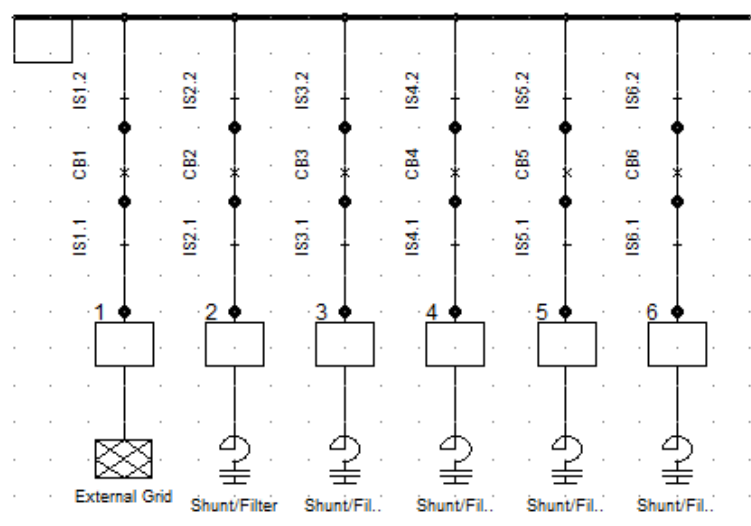

Fig. 13. Grid equivalent at the POI
The source has been connected in series with the first branch, which has no capacitance, and modelled in PowerFactory with the equivalent grid element. The remaining 5 branches have been modelled with an RLC shunt filter element. The values for the parameters of each branch are listed in Table II.

Table II. - Grid equivalent RLC branch parameters

\begin{tabular}{|l|c|c|c|c|c|c|}
\hline & \multicolumn{7}{|c|}{ Equivalent branch number } \\
\hline & 1 & 2 & 3 & 4 & 5 & 6 \\
\hline $\mathrm{R}(\Omega)$ & 1 & 11 & 34.8 & 1.25 & 1.94 & 1.5 \\
\hline $\mathrm{L}(\mathrm{mH})$ & 32.23 & 37.73 & 168.3 & 21.18 & 36.09 & 60.22 \\
\hline $\mathrm{C}(\mu \mathrm{F})$ & & 28.87 & 1.39 & 6.29 & 3.05 & 0.33 \\
\hline
\end{tabular}

Figure 14 shows the harmonic impedance of the reference network (blue curve) and that obtained with the equivalent model (red curve). The harmonic response of the model fits quite well with the real harmonic impedance. The best precision is achieved for the frequency range from $550 \mathrm{~Hz}$ to $1150 \mathrm{~Hz}$, while some precision is lost for lower frequencies. It is not possible to adjust the resistance of the six equivalent branches. It is necessary to select one of them and rescale the rest. For this example, the selection has been made for the resonance frequency of $1069 \mathrm{~Hz}$, which is the one with the highest impedance.

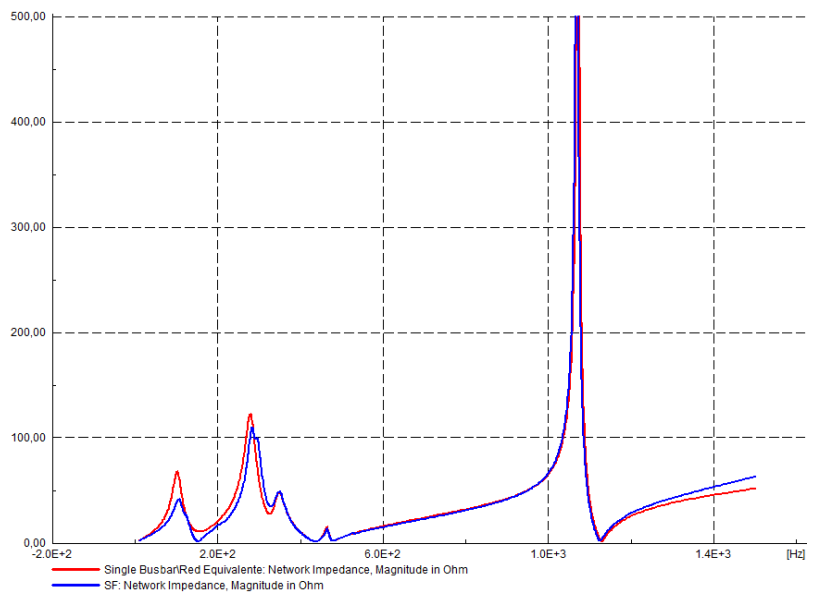

Fig. 14. Comparison equivalent vs complete network model

Finally, a current harmonic injection profile has been assigned to the Onshore HVDC converter and the harmonic voltage has been evaluated at the POI with the complete model and with the equivalent model. The results are compared in Figure 15 with the complete model in blue and the equivalent model in red.

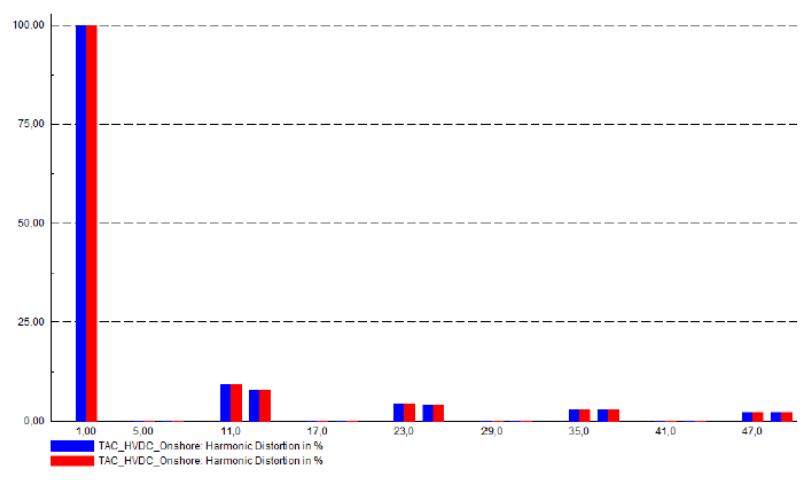

Fig. 15. Comparison equivalent vs complete network model 
Figure 15 shows that the evaluation of the voltage harmonic distortion at the POI provides similar results with both the complete and the equivalent network models.

This example has shown an application case to obtain a network equivalent for a given grid configuration. For other grid configurations, it is necessary to repeat the procedure, starting from the corresponding harmonic impedance profile. The result will be a set of equivalents representing the grid for each operation scenario.

\section{Conclusion}

Harmonic studies for renewable plant connection require to represent the network through a more detailed model than a simple Thevenin equivalent obtained from the short-circuit power at the POI. In this article, different methodologies have been analysed to characterize the harmonic impedance of the network with the aim of obtaining a simple equivalent network model, which can be implemented in any power system simulation software. The method proposed by Hingorani, Narain and Burbery combines precision with simplicity of implementation and provides accurate results when evaluating the harmonic voltage distortion at the POI, as demonstrated in this paper.

\section{Acknowledgement}

The authors thank the support from the Basque Government (project ELKARTEK KK-2017/00083 and research group GISEL ref. IT1083-16) and from the University of the Basque Country UPV/EHU (PPG 17/23).

\section{References}

[1] A. Etxegarai, P. Eguia, E. Torres, G. Buigues and A. Iturregi, "Current procedures and practices on grid code compliance verification of renewable power generation", Renewable and Sustainable Energy Reviews, Vol. 71, May 2017, pp. 191-202.

[2] IEEE PES Wind Plant Collector System Design Working Group, Emin, F. Fernandez, M. Poeller and G. E. Williamson, "Harmonics and Resonance Issues in Wind Power Plants" in Transmission and Distribution Conference and Exposition (T\&D), Orlando, USA, 2012.

[3] IEC 61000-4-7:2002+A1:2008, "Part 4-7: Testing and measurement techniques - General guide on harmonics and interharmonics measurements and instrumentation, for power supply systems and equipment connected thereto", 2008.

[4] S. Gao, X. Li, H. Hu, Z. He, J. Yang, "Measurement-Based Compartmental Modeling of Harmonic Sources in Traction Power-Supply System", IEEE Tran. on PWRD, Vol. 32, No. 2, pp. 900-909, April 2017.

[5] Std. IEC/TR 61000-3-6:2008 "Part 3-6: Limits Assessment of emission limits for the connection of distorting installations to MV, HV and EHV power system", 2008

[6] Std. 519-1992 - IEEE Recommended Practices and Requirements for Harmonic Control in Electrical Power Systems.

[7] Z Emin, F Fernandez, M Poeller, G E Williamson, "Harmonic Distortion Specification and Compliance of an Offshore Wind Generation", in $10^{\text {th }}$ IET International Conference on AC and DC Power Transmission, Birmingham, UK, 2012.

[8] A. S. Morched, J. H. Ottevangers, L. Martí, "Multi-Port Frequency Dependent Network Equivalents for the EMTP", 1993.

[9] DIgSILENT PowerFactory Tutorial, 2017.

[10] Hingorani, Narain G., and Michael F. Burbery. "Simulation of AC system impedance in HVDC system studies." IEEE Transactions on power Apparatus and Systems 5 (1970): 820-828.

[11] DIgSILENT GmbH Training, "360 MW Offshore Wind Farm Atlantis", 2017 